\title{
Confirming a Cosmic-Ray Bump
}

\author{
The DArk Matter Particle Explorer has made the most precise \\ measurements of galactic cosmic rays to date.
}

By Katherine Wright

$\mathrm{F}$

or over 5 years, the DArk Matter Particle Explorer

(DAMPE) has orbited Earth measuring cosmic rays. The team behind the telescope has now analyzed 4.5 years of cosmic-ray data, finding spectral features that don't match predictions [1]. While similar features were hinted at in other experiments, the measurements by DAMPE have a higher precision and cover a wider range of energies than any other single experiment. The findings could help researchers uncover the origin of galactic cosmic rays.

Cosmic rays consist mostly of protons and helium ions and are thought to emanate from supernovae. On their journey to Earth the rays are deflected by interstellar magnetic fields, making it hard to determine their sources. But researchers hope that by measuring the energy spectra of cosmic rays, they can extract some information about the supernovae that sent them flying and about the structure of our Galaxy.

In their analysis, the DAMPE team analyzed the energy spectrum of detected helium ions. These particles had energies from 70 $\mathrm{GeV}$ to $80 \mathrm{TeV}$, an order of magnitude higher than those detected with the Alpha Magnetic Spectrometer aboard the International

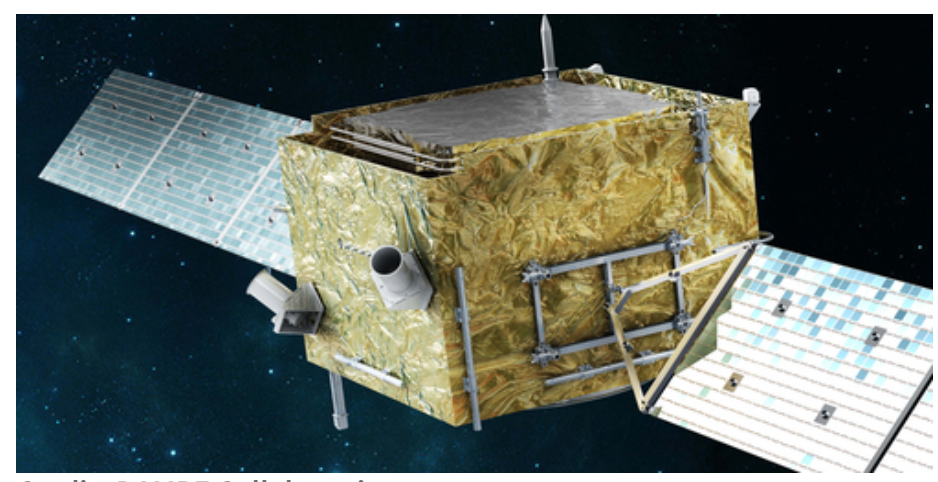

Credit: DAMPE Collaboration

Space Station (see Focus: New Data Reveal the Heavy Side of Cosmic Rays) and 100 times higher than those seen with the PAMELA satellite (see Synopsis: Solar Cycle Affects Cosmic Ray Positrons). At around 1.3 TeV the team observed the intensity of the spectrum start to rise, peaking at about $34 \mathrm{TeV}$. The statistical significance of the finding is 4.3 sigma. Signs of such a bump have been seen before, but the uncertainties in previous data were too large to confirm the bump's presence. The team says that they think the bump-like feature might be caused by a nearby supernova, but that remains unconfirmed.

Katherine Wright is the Deputy Editor of Physics.

\section{REFERENCES}

1. F. Alemanno et al., "Measurement of the cosmic ray helium energy spectrum from $70 \mathrm{GeV}$ to $80 \mathrm{TeV}$ with the DAMPE space mission," Phys. Rev. Lett. 126, 201102 (2021). 